\title{
Direct detection of light dark matter from evaporating primordial black holes
}

\author{
Roberta Calabrese $\odot,{ }^{1,2}$ Marco Chianese $\odot,{ }^{1,2, *}$ Damiano F. G. Fiorillo, ${ }^{1,2}$ and Ninetta Saviano $\circledast^{2,3}$ \\ ${ }^{1}$ Dipartimento di Fisica "Ettore Pancini", Università degli studi di Napoli "Federico II", \\ Complesso Universitario Monte S. Angelo, I-80126 Napoli, Italy \\ ${ }^{2}$ INFN_Sezione di Napoli, Complesso Universitario Monte S. Angelo, I-80126 Napoli, Italy \\ ${ }^{3}$ Scuola Superiore Meridionale, Università degli studi di Napoli "Federico II", \\ Largo San Marcellino 10, 80138 Napoli, Italy
}

(Received 16 August 2021; revised 9 October 2021; accepted 8 December 2021; published 5 January 2022)

\begin{abstract}
The direct detection of sub-GeV dark matter interacting with nucleons is hampered by the low recoil energies induced by scatterings in the detectors. This experimental difficulty is avoided in the scenario of boosted dark matter where a component of dark matter particles is endowed with large kinetic energies. In this paper, we point out that the current evaporation of primordial black holes with a mass from $10^{14}$ to $10^{16} \mathrm{~g}$ is a source of boosted light dark matter with energies of tens to hundreds of MeV. Focusing on the XENON1T experiment, we show that these relativistic dark matter particles could give rise to a signal orders of magnitude larger than the present upper bounds. Therefore, we are able to significantly constrain the combined parameter space of primordial black holes and sub-GeV dark matter. In the presence of primordial black holes with masses of $10^{15} \mathrm{~g}$ and abundances compatible with present bounds, the limits on a dark matter-nucleon cross section are improved by four orders of magnitude.
\end{abstract}

DOI: 10.1103/PhysRevD.105.L021302

\section{INTRODUCTION}

Although dark matter (DM) is one of the backbones of the standard cosmological model, its nature is still unknown [1]. So far, all the evidences of its existence are related only to its gravitational imprints, while we have no clue about its nongravitational interactions [2,3]. Among the different ways to probe DM properties, direct detection experiments are achieving very stringent constraints on DM-nucleon [4-19] and DM-electron [20-26] interactions (see Ref. [27] for a recent review). These experiments search for the nuclear and electron recoil energy caused by the possible scatterings with DM particles that surround us. Due to a rapidly decreasing sensitivity at low recoil energies, the constraints on DM interactions dramatically weaken for DM masses smaller than about $1 \mathrm{GeV}(1 \mathrm{MeV})$ for DM interactions with nuclei (electrons), thus leaving light DM candidates poorly explored by direct searches. There are complementary approaches to the exploration of light DM. Fermionic DM particles lighter than $0.1 \mathrm{keV}$ are highly constrained by phase-space

\footnotetext{
chianese@na.infn.it
}

Published by the American Physical Society under the terms of the Creative Commons Attribution 4.0 International license. Further distribution of this work must maintain attribution to the author(s) and the published article's title, journal citation, and DOI. Funded by SCOAP. arguments [28,29] and structure formation [30]. Other model-dependent constraints are placed by astrophysical and cosmological observations, as well as by colliders [31-40].

The experimental limitation of direct detection can be circumvented in the framework of boosted dark matter, where a fraction of DM particles gains a velocity higher than the virial one due to a number of different mechanisms [41-43]. In recent years, it has been pointed out that light DM particles can be upscattered to (semi)relativistic velocities through collisions with cosmic rays [44-49]. The unavoidable presence of such a subdominant boosted DM component has improved the model-independent constraints on the DM-nucleon cross section at the level of $\sim 10^{-31} \mathrm{~cm}^{2}$.

In this letter, we propose the evaporation of primordial black holes (PBHs) at present times as a source of boosted light DM particles (hereafter denoted as $\chi$ ). Throughout this work, we refer to this scenario as ePBH-DM. PBHs are hypothetical black holes formed soon after the inflationary epoch through the gravitational collapse of density fluctuations in the early Universe [50,51]. As a result of combining quantum field theory and general relativity, PBHs emit Hawking radiation in the form of the graybody spectrum with a temperature $T_{\mathrm{PBH}}$ inversely proportional to the PBH mass [52-58]. $\mathrm{PBHs}$ with a mass $M_{\mathrm{PBH}}=$ $\mathcal{O}\left(10^{15} \mathrm{~g}\right)$ are evaporating at the present times, a process that has been employed to set stringent upper bounds on 
the PBHs abundance [59-66] (see Ref. [67] for a comprehensive review). DM particles with a mass $m_{\chi}<T_{\mathrm{PBH}}$ are also emitted with (semi)relativistic momenta by PBHs. This mechanism has been vastly studied as a way to produce DM particles in the early Universe [68-77]. However, the production of DM particles in the present Universe, to our knowledge, has never been investigated.

Here, we explore for the first time the phenomenological implications of the ePBH-DM scenario in direct detection experiments. Remarkably, we find that even a tiny fraction of evaporating PBHs is enough to give rise to a sizeable flux of boosted light DM particles (see Fig. 1). This translates into a detectable event rate in current experiments such as XENON1T in case DM particles interact with nucleons (see Fig. 2). Hence, the existence of PBHs would imply incredibly strong constraints on the DM parameter space, and vice versa, as shown in Fig. 3. We find that, assuming PBHs abundances compatible with current bounds, the limits on the spin-independent (SI) DMnucleon cross section are improved up to four orders of magnitude. Conversely, the nonobservation of the ePBHDM signal allows us to deduce upper bounds on the PBHs abundance a few orders of magnitude stronger than current constraints, depending on the strength of DM-nucleon interactions. We remark that our constraints do not require the $\chi$ particles to be the dominant DM component.

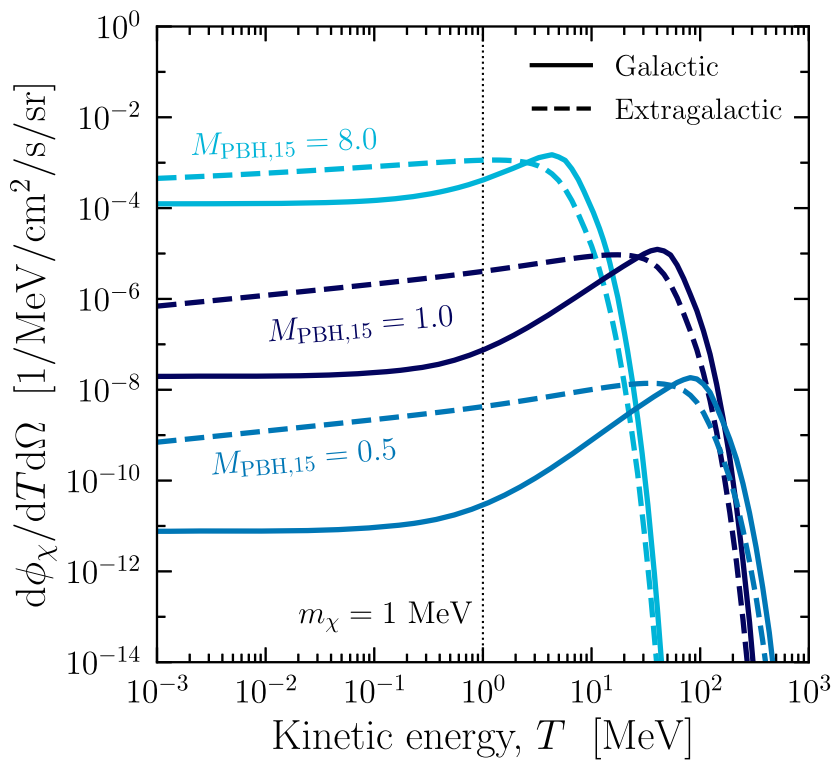

FIG. 1. Diffuse DM flux from evaporating PBHs. We show the diffuse flux of DM particles incident at the Earth as a function of the kinetic energy. The Galactic (solid) and extragalactic (dashed) lines are shown separately. Different colors are used to identify different $\mathrm{PBH}$ masses $\left(M_{\mathrm{PBH}, 15}=M_{\mathrm{PBH}} / 10^{15} \mathrm{~g}\right)$. The $\mathrm{PBH}$ abundance is chosen as the maximum value allowed by the present constraints [67]; $f_{\mathrm{PBH}}$ is equal to $2.9 \times 10^{-10}, 3.9 \times 10^{-7}$, and $3.7 \times 10^{-4}$ for a PBH mass of $0.5,1.0$, and 8.0 in units of $10^{15} \mathrm{~g}$, respectively. Effects of energy losses in Earth and atmosphere are not included.

\section{A. DM flux from evaporating PBHs}

The first step of our work consists of obtaining the DM emission rate from an evaporating $\mathrm{PBH}$. We here make a conservative choice by considering chargeless and spinless PBHs. Spinning PBHs would evaporate faster, thus enhancing the DM emission rate. For the sake of concreteness, we examine the case of DM Dirac fermions with four degrees of freedom, $g_{\chi}=4$. However, this framework can be easily extended to scalar and vector DM particles. Differently from Ref. [66], the addition of only one new species to the Standard Model particle content does not significantly alter the standard emission rate of the Hawking radiation. The Hawking temperature of an evaporating $\mathrm{PBH}$ with mass $M_{\mathrm{PBH}}$ is given by $[53,56-58]$

$$
k_{\mathrm{B}} T_{\mathrm{PBH}}=10.6\left[\frac{10^{15} \mathrm{~g}}{M_{\mathrm{PBH}}}\right] \mathrm{MeV}
$$

where $k_{\mathrm{B}}$ is the Boltzmann constant. The differential spectrum per unit time is

$$
\frac{\mathrm{d} N_{\chi}}{\mathrm{d} T \mathrm{~d} t}=\frac{g_{\chi}}{2 \pi} \frac{\Gamma\left(T, M_{\mathrm{PBH}}\right)}{\exp \left[\left(T+m_{\chi}\right) / k_{\mathrm{B}} T_{\mathrm{PBH}}\right]+1},
$$

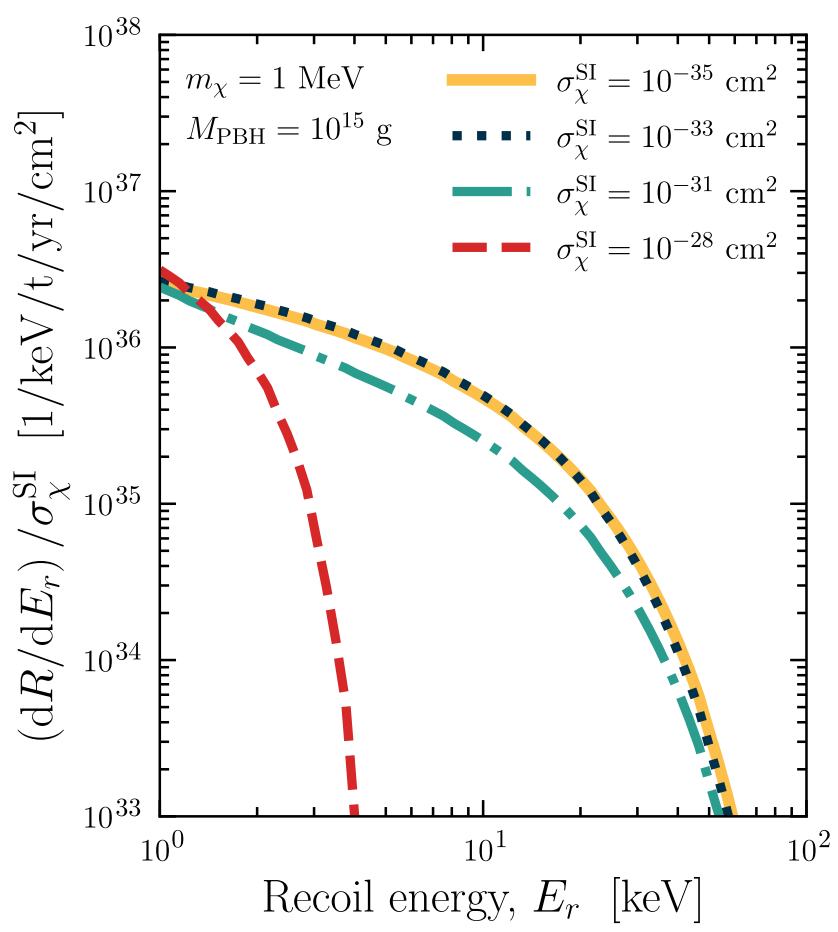

FIG. 2. Ratio of XENON1T event rate over DM-nucleon cross section. We show the ratio of the event rate induced by DM scatterings inside the XENON1T detector over the DM-nucleon cross section. Here, we account for the effects of propagation through the Earth and the atmosphere. The lines correspond to different values of the DM-nucleon cross section. The remaining parameters are chosen as $M_{\mathrm{PBH}}=10^{15} \mathrm{~g}, f_{\mathrm{PBH}}=3.9 \times 10^{-7}$, and $m_{\chi}=1 \mathrm{MeV}$. 

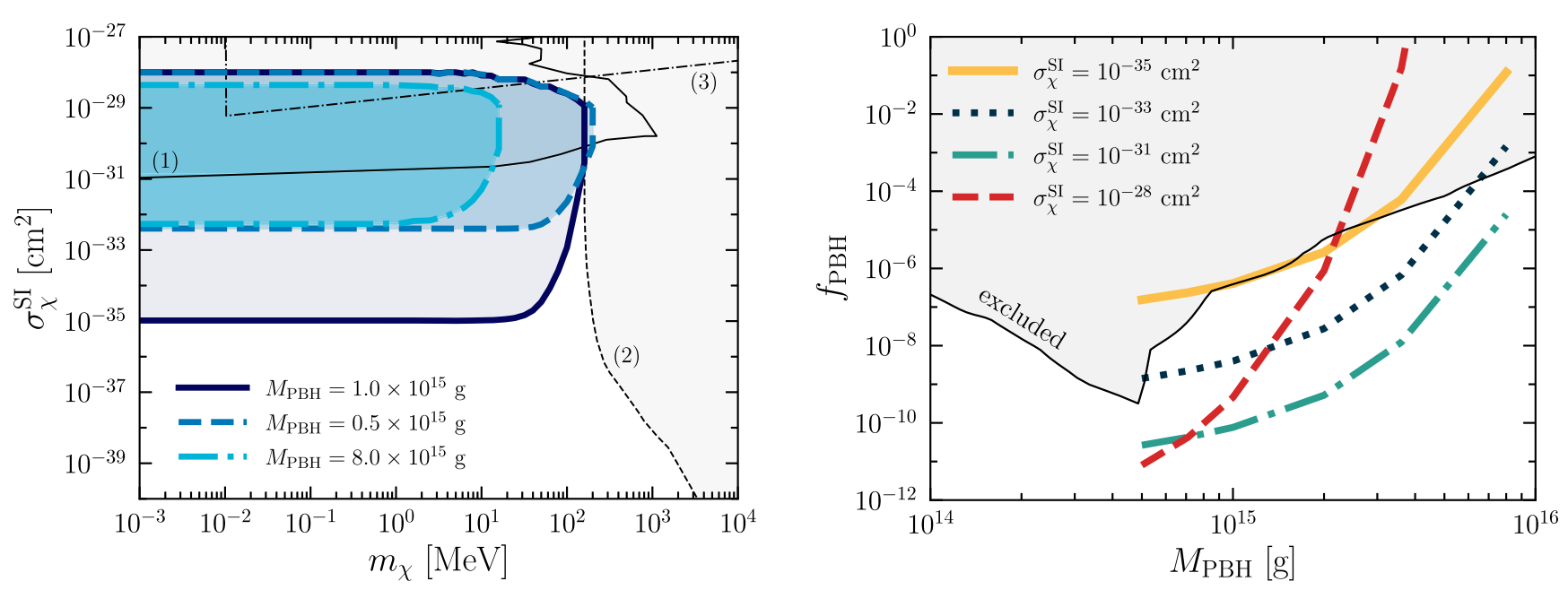

FIG. 3. Constraints on the ePBH-DM parameter space. We show the regions excluded by the XENON1T experiment in the planes $m_{\chi}-\sigma_{\chi}^{\mathrm{SI}}$ (left) and $M_{\mathrm{PBH}}-f_{\mathrm{PBH}}$ (right). In the left panel, the excluded regions are obtained for different values of $M_{\mathrm{PBH}}$; for each case, $f_{\mathrm{PBH}}$ is set to the maximum value allowed by present constraints [67] (also shown with the thin black line in the right plot). For comparison, we also show previous DM constraints from cosmic-ray boosted DM particles [45,47] (1), from the CRESST experiment $[15,16](2)$, and from cosmology [37-40] (3). In the right panel, the different thick colored lines correspond to the upper bound on $f_{\mathrm{PBH}}$ obtained for different DM-nucleon cross sections and choosing $m_{\chi}$ smaller than $1 \mathrm{MeV}$.

where $T$ is the kinetic energy of the particle, and $\Gamma$ is the graybody factor, which we compute by means of the BlackHawk code [78].

From the differential spectrum, we can compute the flux of DM particles reaching the Earth for a monochromatic PBH mass distribution. It consists of a Galactic (gal) and an extragalactic (egal) component,

$$
\frac{\mathrm{d} \phi_{\chi}}{\mathrm{d} T \mathrm{~d} \Omega}=\frac{\mathrm{d} \phi_{\chi}^{\mathrm{gal}}}{\mathrm{d} T \mathrm{~d} \Omega}+\frac{\mathrm{d} \phi_{\chi}^{\text {egal }}}{\mathrm{d} T \mathrm{~d} \Omega} .
$$

The first component can be written as

$$
\frac{\mathrm{d} \phi_{\chi}^{\mathrm{gal}}}{\mathrm{d} T \mathrm{~d} \Omega}=\frac{f_{\mathrm{PBH}}}{4 \pi M_{\mathrm{PBH}}} \frac{\mathrm{d} N_{\chi}}{\mathrm{d} T \mathrm{~d} t} \int_{0}^{+\infty} \mathrm{d} s \rho_{\mathrm{DM}}^{\mathrm{NFW}}(r) .
$$

Here, the quantity $f_{\mathrm{PBH}}=\rho_{\mathrm{PBH}} / \rho_{\mathrm{DM}}$ is the fraction of PBHs with respect to the average DM density $\rho_{\mathrm{DM}}$ of the Universe, which is determined by Planck [79]. The Galactic flux is proportional to the integral over the line-of-sight distance $s$ of the Galactic DM density, denoted as $\rho_{\mathrm{DM}}^{\mathrm{NFW}}$, for which we assume a Navarro-Frank-White profile [80]. This is a function of the galactocentric distance $r=\left(r_{\odot}^{2}+\right.$ $\left.s^{2}-2 s r_{\odot} \cos \ell \cos b\right)^{1 / 2}$ with $r_{\odot}=8.5 \mathrm{kpc}$ and $(b, \ell)$ the Galactic coordinates.

For the extragalactic component in Eq. (3), the differential flux takes the expression

$$
\frac{\mathrm{d} \phi_{\chi}^{\text {egal }}}{\mathrm{d} T \mathrm{~d} \Omega}=\frac{f_{\mathrm{PBH}} \rho_{\mathrm{DM}}}{4 \pi M_{\mathrm{PBH}}} \int_{t_{\min }}^{t_{\max }} \mathrm{d} t[1+z(t)] \frac{\mathrm{d} N_{\chi}}{\mathrm{d} T \mathrm{~d} t},
$$

where we take into account the effect of redshift $z(t)$ on the energy in the DM emission rate, and we integrate from the time of matter-radiation equality $\left(t_{\min }\right)$ to the age of the Universe $\left(t_{\max }\right)$. Differently from the Galactic component, which is enhanced towards the Galactic center, the extragalactic DM flux is isotropic.

Given the evaporation temperatures of $\mathrm{PBHs}$ from Eq. (1), DM particles are mainly produced at energies between 1 and $100 \mathrm{MeV}$, for PBHs masses from $10^{14}$ and to $10^{16} \mathrm{~g}$. Hence, DM particles lighter than about $1 \mathrm{MeV}$ are emitted by PBHs with ultrarelativistic velocities. In a sense, the evaporation of PBHs is a new mechanism for boosted DM. In Fig. 1, we show the Galactic and extragalactic diffuse flux of DM particles at the Earth, averaged over the whole solid angle, for a reference DM mass $m_{\chi}=1 \mathrm{MeV}$ and for different PBH masses. The kinetic energy distribution of particles peaks at different energies depend on the $\mathrm{PBH}$ temperature, which can be much larger than the DM mass. For kinetic energies much smaller than the PBH mass, the Galactic spectrum flattens since the particles are produced practically at rest. The normalization of the flux is mainly determined by the PBH mass and the concentration of PBHs $f_{\mathrm{PBH}}$. Larger PBH masses lead to slower evaporation rates and lower fluxes. However, at larger $\mathrm{PBH}$ masses, the constraints on $f_{\mathrm{PBH}}$ are also weaker (see the right plot in Fig. 3), leading to larger fluxes in Fig. 1.

\section{B. Propagation through Earth and atmosphere}

Direct detection experiments probe DM particles through their potential interactions with nucleons. These processes, however, might also cause an attenuation of the 
DM flux at the detector position due to the propagation in the Earth and in the atmosphere [81-84]. For the sake of simplicity, we here model this phenomenon by following the analytical approach described in Ref. [45]. In particular, we employ a ballistic-trajectory approximation and compute the energy loss of DM particles reaching the detector. Since the kinetic energy is typically $T \lesssim 100 \mathrm{MeV}$, we can neglect quasielastic and inelastic scatterings as well as the nuclear form factors. Under this assumption, the interaction lengths in the Earth $\left(\ell_{\text {int }}^{\oplus}\right)$ and in the atmosphere $\left(\ell_{\text {int }}^{\text {atm }}\right)$ read as

$$
\ell_{\mathrm{int}}^{i}=\left[\sum_{N} n_{\mathrm{N}}^{i} \sigma_{\chi \mathrm{N}} \frac{2 m_{\mathrm{N}} m_{\chi}}{\left(m_{\mathrm{N}}+m_{\chi}\right)^{2}}\right]^{-1},
$$

where the sum, respectively, runs over the most abundant elements in the Earth (from iron to aluminium) and the atmosphere (nitrogen and oxygen) with number density $n_{\mathrm{N}}^{i}$, whereas the cross section is

$$
\sigma_{\chi \mathrm{N}}=\sigma_{\chi}^{\mathrm{SI}} \mathrm{A}^{2}\left(\frac{m_{\mathrm{N}}\left(m_{\chi}+m_{\mathrm{p}}\right)}{m_{\mathrm{p}}\left(m_{\chi}+m_{\mathrm{N}}\right)}\right)^{2} .
$$

Here, we focus on the spin-independent scatterings which feature the standard coherent enhancement with the mass number A. Moreover, we limit ourselves to the case of a isospin-singlet structure assuming it equals the DM interaction with protons and neutrons. In this framework, DM particles with an initial kinetic energy $T_{0}$ reach the detector with a smaller kinetic energy $T_{d}$ after traveling a total geometrical distance $d=d_{\mathrm{atm}}+d_{\oplus}$. We take into account that the xenon1T detector is located in the Gran Sasso National Laboratory at a depth of about $1.4 \mathrm{~km}$ [85]. Hence, the actual DM flux reaching the XENON1T detector can be obtained as

$$
\frac{\mathrm{d} \phi_{\chi}^{d}}{\mathrm{~d} T_{d} \mathrm{~d} \Omega} \approx \frac{4 m_{\chi}^{2} e^{\tau}}{\left(2 m_{\chi}+T_{d}-T_{d} e^{\tau}\right)^{2}}\left(\left.\frac{\mathrm{d} \phi_{\chi}}{\mathrm{d} T \mathrm{~d} \Omega}\right|_{T_{0}}\right)
$$

where $\tau=d_{\mathrm{atm}} / \ell_{\mathrm{int}}^{\mathrm{atm}}+d_{\oplus} / \ell_{\mathrm{int}}^{\oplus}$, and the incoming DM flux is evaluated at

$$
T_{0}\left(T_{d}\right)=\frac{2 m_{\chi} T_{d} e^{\tau}}{2 m_{\chi}+T_{d}-T_{d} e^{\tau}} .
$$

For a given incoming direction defined by the Galactic coordinates $(b, \ell)$, the distance $d$ depends on the timedependent longitude of the experiment. Thus, in the following, we consider the average of Eq. (8) over a day.

In closing this section, we may stress that the approach of Ref. [45] is based on the approximation of collinear, or ballistic, propagation of DM in matter. As pointed out in Ref. [47], this approximation does not apply to the collisions of light DM with heavy nuclei; rather, the propagation of DM happens in a diffusive way, leading to a larger energy loss and a possible reflection of DM from the Earth and the atmosphere. Such a more complicated propagation might lower the ePBH-DM flux detectable at the experiment. In order to understand the impact of the ballistic approximation, we have computed the flux under the most conservative assumption that particles are completely depleted by the scattering (in other words, particles which have undergone even a single scattering are not considered). Even under this extreme assumption, we still find large DM fluxes observable in underground experiments. Therefore, we expect that a more realistic treatment of the DM propagation should not disrupt the scenario presented here. As is shown later, the ePBH-DM scenario allows us to probe a DM-nucleon cross section at the level of $10^{-35} \mathrm{~cm}^{2}$, for which the effects of propagation are marginal, since the atmosphere is completely transparent to DM.

\section{XENON1T event rate}

We can now compute the event rate expected in the XENON1T detector in the ePBH-DM scenario. In this experiment, the main target for DM scatterings is provided by xenon nuclei. The differential event rate (number of events per ton year) per xenon recoil energy $E_{r}$ can be obtained as

$\frac{\mathrm{d} R}{\mathrm{~d} E_{r}}=\sigma_{\chi \mathrm{Xe}} \mathcal{N}_{\text {Xe }} \int \mathrm{d} T_{d} \mathrm{~d} \Omega \frac{\mathrm{d} \phi_{\chi}^{d}}{\mathrm{~d} T_{d} \mathrm{~d} \Omega} \frac{\Theta\left(E_{r}^{\max }-E_{r}\right)}{E_{r}^{\max }}$,

where $\mathcal{N}_{\mathrm{Xe}}$ is the total number of targets, and

$$
E_{r}^{\max }=\frac{T_{d}^{2}+2 m_{\chi} T_{d}}{T_{d}+\left(m_{\chi}+m_{\mathrm{Xe}}\right)^{2} /\left(2 m_{\mathrm{Xe}}\right)}
$$

is the maximum allowed value for the recoil energy, for which we assume a flat distribution. In this estimate, the cross section $\sigma_{\chi \mathrm{Xe}}$ includes the corresponding nuclear form factor as provided in Refs. [86,87]. Moreover, we consider an exposure of one ton year.

The event rate at the XENON1T detector is shown in Fig. 2 as a function of the recoil energy. In order to highlight the effect of propagation through the Earth and the atmosphere, we report the ratio of the event rate and the DM-nucleon cross section $\sigma_{\chi}^{\text {SI }}$. In the absence of propagation effects, this ratio would be independent of the cross section. This can be seen in the case of the solid yellow and dotted blue curve (corresponding to low cross sections), which are practically identical. For $\sigma_{\chi}^{S I}=10^{-31} \mathrm{~cm}^{2}$ and $\sigma_{\chi}^{\mathrm{SI}}=10^{-28} \mathrm{~cm}^{2}$, the effects of propagation are more evident, pushing the events to lower recoil energies and weakening the sensitivity to the ePBH-DM signal. 


\section{Results}

In order to probe the ePBH-DM scenario, we need to compare the expected event rate with the current data from XENON1T [11]. This experiment has observed no excess of recoil events in the energy window from $E_{r, 1}=4.9 \mathrm{keV}$ to $E_{r, 2}=40.9 \mathrm{keV}$. Following Ref. [45], this can be translated into an upper limit on the total event rate as

$\int_{E_{r, 1}}^{E_{r, 2}} \mathrm{~d} E_{r} \frac{\mathrm{d} R}{\mathrm{~d} E_{r}} \lesssim \kappa \bar{v}_{\mathrm{DM}} \rho_{\mathrm{DM}}^{\odot} \mathcal{N}_{\mathrm{Xe}}\left(\frac{\sigma_{\chi \mathrm{Xe}}}{m_{\chi}}\right)_{m_{\chi} \gtrsim 100 \mathrm{GeV}}$,

where $\kappa \simeq 0.23, \bar{v}_{\mathrm{DM}} \simeq 235 \mathrm{~km} / \mathrm{s}$ is the mean DM velocity in the usual scenario, $\rho_{\mathrm{DM}}^{\odot}=0.3 \mathrm{GeV} / \mathrm{cm}^{3}$ the local DM density as assumed by XENON1T Collaboration, and the term in parentheses $2.5 \times 10^{-40} \mathrm{~cm}^{2} / \mathrm{GeV}$ according to the current XENON1T limit for large DM masses [11].

Equation (12) translates into the limits on the combined parameter space of DM and PBHs shown in Fig. 3. In particular, the left plot shows the regions of the DM parameter space that are constrained by XENON1T, in the case of three different PBH masses. For each PBH mass, we consider the maximum allowed value for $f_{\mathrm{PBH}}$ in agreement with current limits [67]. The gray region is excluded by previous constraints on sub-GeV DM particles from cosmic-ray upscatterings [45,47], CRESST experiment $[15,16]$, and cosmology [37-40]. Here, we only focus on the region $m_{\chi} \geq 10^{-3} \mathrm{MeV}$ since lighter fermionic DM is highly disfavored. However, our constraints virtually extend to lower DM masses. For $m_{\chi}=1 \mathrm{MeV}$, we have checked that the DM constraints are unchanged for complex scalar DM particles, while they weaken by about $80 \%$ for vector DM particles.

In the right plot, we report the upper bounds on the PBH abundance as a function of the PBH mass, assuming four benchmark values for the DM-nucleon cross section. Here, we consider DM masses smaller than $1 \mathrm{MeV}$. For low DM-nucleon cross sections, the constraints on $f_{\mathrm{PBH}}$ are almost inversely proportional to $\sigma_{\chi}^{\mathrm{SI}}$. For $\sigma_{\chi}^{\mathrm{SI}}=10^{-28} \mathrm{~cm}^{-2}$, instead, the peculiar behavior of the constraint is due to the considerable effects of the propagation in the Earth and the atmosphere. We emphasize that our constraints are valid for $0.5 \lesssim M_{\mathrm{PBH}} /\left(10^{15} \mathrm{~g}\right) \lesssim 8$.0. Lighter PBHs are almost completely evaporated, whereas heavier ones emit DM particles with too low kinetic energies.

\section{E. Discussion}

The ePBH-DM scenario with PBH masses from $10^{14}$ to $10^{16} \mathrm{~g}$ leads to signatures that are already testable with direct detection experiments. The constraints presented in this work apply to any model with the simultaneous presence of PBHs and DM coupled to nucleons. For low DM-nucleon cross sections, our results mainly depend on the spectrum of DM from PBHs and therefore are expected to be robust. For large cross sections, the different approaches proposed in the literature to describe the DM propagation through Earth and the atmosphere might lead to slightly different results. However, this does not influence the possibility of constraining the scenario under consideration.

Since PBHs constitute only a very small fraction of the total energy of the nonrelativistic DM in the Universe, the emitted DM particles are expected to not change significantly the properties of the Galactic DM distribution and the extragalactic DM density. Indeed, the magnitude of the signal is not connected with a particularly large DM flux from PBHs but rather with the poorly constrained DMnucleon cross sections for sub-GeV DM. Our work shows that cross sections from $10^{-35}$ to $10^{-31} \mathrm{~cm}^{2}$, while allowed by present constraints, may be inconsistent with the existence of PBHs evaporating at present times.

We emphasize that there is a strong degeneracy between the amount of PBHs and the strength of the DM interaction with nucleons. For low cross sections, where the effects of propagation are negligible, the ePBH-DM signal is indeed proportional to the product $f_{\mathrm{PBH}} \cdot \sigma_{\chi}^{\mathrm{SI}}$. Future observations of evaporating PBHs, for example, through the gamma-ray and neutrino burst produced in a PBH evaporation [88-95], might confirm their existence and remove this degeneracy, allowing for definite constraints on the DM-nucleon interaction.

\section{ACKNOWLEDGMENTS}

We thank Gennaro Miele and Stefano Morisi for useful comments and discussions. This work was supported by the research Grant No. 2017W4HA7S "NAT-NET: Neutrino and Astroparticle Theory Network" under the program PRIN 2017 funded by the Italian Ministero dell'Università e della Ricerca (MUR) and by the research project TAsP (Theoretical Astroparticle Physics) funded by the Istituto Nazionale di Fisica Nucleare Sezione di Napoli.
[1] G. Bertone and M. P. T. Tait, A new era in the search for dark matter, Nature (London) 562, 51 (2018).

[2] F. Kahlhoefer, Review of LHC dark matter searches, Int. J. Mod. Phys. A 32, 1730006 (2017).
[3] C. Pérez de los Heros, Status, challenges and directions in indirect dark matter searches, Symmetry 12, 1648 (2020).

[4] G. Angloher, C. Bucci, P. Christ, C. Cozzini, F. von Feilitzsch, D. Hauff, S. Henry, Th. Jagemann, J. Jochum, 
H. Kraus et al., Limits on wimp dark matter using scintillating $\mathrm{CaWO}_{4}$ cryogenic detectors with active background suppression, Astropart. Phys. 23, 325 (2005).

[5] E. Aprile, M. Alfonsi, K. Arisaka, F. Arneodo, C. Balan, L. Baudis, B. Bauermeister, A. Behrens, P. Beltrame, K. Bokeloh et al. (XENON100 Collaboration), Dark Matter Results from 225 Live Days of XENON100 Data, Phys. Rev. Lett. 109, 181301 (2012).

[6] P. Agnes et al. (DarkSide Collaboration), First results from the DarkSide-50 dark matter experiment at Laboratori Nazionali del Gran Sasso, Phys. Lett. B 743, 456 (2015).

[7] G. Angloher et al., The COSINUS project-perspectives of a NaI scintillating calorimeter for dark matter search, Eur. Phys. J. C 76, 441 (2016).

[8] J. Aalbers et al. (DARWIN Collaboration), DARWIN: Towards the ultimate dark matter detector, J. Cosmol. Astropart. Phys. 11 (2016) 017.

[9] R. Agnese et al. (SuperCDMS Collaboration), Projected sensitivity of the SuperCDMS SNOLAB experiment, Phys. Rev. D 95, 082002 (2017).

[10] C. E. Aalseth et al. (DarkSide-20k Collaboration), DarkSide-20k: A 20 tonne two-phase LAr TPC for direct dark matter detection at LNGS, Eur. Phys. J. Plus 133, 131 (2018).

[11] E. Aprile et al. (XENON Collaboration), Dark Matter Search Results from a One Ton-Year Exposure of XENON1T, Phys. Rev. Lett. 121, 111302 (2018).

[12] D. S. Akerib et al. (LUX-ZEPLIN Collaboration), Projected WIMP sensitivity of the LUX-ZEPLIN dark matter experiment, Phys. Rev. D 101, 052002 (2020).

[13] E. Aprile et al. (XENON Collaboration), Light Dark Matter Search with Ionization Signals in XENON1T, Phys. Rev. Lett. 123, 251801 (2019).

[14] E. Aprile et al. (XENON Collaboration), Search for Light Dark Matter Interactions Enhanced by the Migdal Effect or Bremsstrahlung in XENON1T, Phys. Rev. Lett. 123, 241803 (2019).

[15] G. Angloher et al. (CRESST Collaboration), Results on $\mathrm{MeV}$-scale dark matter from a gram-scale cryogenic calorimeter operated above ground, Eur. Phys. J. C 77, 637 (2017).

[16] A. H. Abdelhameed et al. (CRESST Collaboration), First results from the CRESST-III low-mass dark matter program, Phys. Rev. D 100, 102002 (2019).

[17] E. Aprile et al. (XENON Collaboration), Projected WIMP sensitivity of the XENONnT dark matter experiment, J. Cosmol. Astropart. Phys. 11 (2020) 031.

[18] G. Adhikari et al. (COSINE-100 Collaboration), Strong constraints from COSINE-100 on the DAMA dark matter results using the same sodium iodide target, Sci. Adv. 7, abk2699 (2021).

[19] Y. Meng et al. (PandaX Collaboration), Dark Matter Search Results from the PandaX-4T Commissioning Run, Phys. Rev. Lett. 128, 261802 (2022).

[20] R. Essig, J. Mardon, and T. Volansky, Direct detection of sub-GeV dark matter, Phys. Rev. D 85, 076007 (2012).

[21] R. Essig, A. Manalaysay, J. Mardon, P. Sorensen, and T. Volansky, First Direct Detection Limits on sub-GeV Dark Matter from XENON10, Phys. Rev. Lett. 109, 021301 (2012).
[22] R. Essig, T. Volansky, and T.-T. Yu, New constraints and prospects for sub-GeV dark matter scattering off electrons in xenon, Phys. Rev. D 96, 043017 (2017).

[23] M. Crisler, R. Essig, J. Estrada, G. Fernandez, J. Tiffenberg, M. Sofo haro, T. Volansky, and T.-T. Yu (SENSEI Collaboration), SENSEI: First Direct-Detection Constraints on sub-GeV Dark Matter from a Surface Run, Phys. Rev. Lett. 121, 061803 (2018).

[24] P. Agnes et al. (DarkSide Collaboration), Constraints on Sub-GeV Dark-Matter-Electron Scattering from the DarkSide-50 Experiment, Phys. Rev. Lett. 121, 111303 (2018).

[25] A. Aguilar-Arevalo et al. (DAMIC Collaboration), Constraints on Light Dark Matter Particles Interacting with Electrons from DAMIC at SNOLAB, Phys. Rev. Lett. 123, 181802 (2019).

[26] E. Andersson, A. Bökmark, R. Catena, T. Emken, H. Klein Moberg, and E. Åstrand, Projected sensitivity to sub-GeV dark matter of next-generation semiconductor detectors, J. Cosmol. Astropart. Phys. 05 (2020) 036.

[27] M. Schumann, Direct detection of WIMP dark matter: Concepts and status, J. Phys. G 46, 103003 (2019).

[28] A. Boyarsky, O. Ruchayskiy, and D. Iakubovskyi, A lower bound on the mass of dark matter particles, J. Cosmol. Astropart. Phys. 03 (2009) 005.

[29] C. Di Paolo, F. Nesti, and F. L. Villante, Phase space mass bound for fermionic dark matter from dwarf spheroidal galaxies, Mon. Not. R. Astron. Soc. 475, 5385 (2018).

[30] S. Tremaine and J.E. Gunn, Dynamical Role of Light Neutral Leptons in Cosmology, Phys. Rev. Lett. 42, 407 (1979).

[31] T. Cohen, M. Lisanti, and H. Keong Lou, Semivisible Jets: Dark Matter Undercover at the LHC, Phys. Rev. Lett. 115, 171804 (2015).

[32] N. Daci, Isabelle De Bruyn, S. Lowette, M. H. G. Tytgat, and B. Zaldivar, Simplified SIMPs and the LHC, J. High Energy Phys. 11 (2015) 108.

[33] Y. Ali-Haïmoud, J. Chluba, and M. Kamionkowski, Constraints on Dark Matter Interactions with Standard Model Particles from Cosmic Microwave Background Spectral Distortions, Phys. Rev. Lett. 115, 071304 (2015).

[34] M. Baryakhtar, J. Bramante, S. Weishi Li, T. Linden, and N. Raj, Dark Kinetic Heating of Neutron Stars and An Infrared Window On WIMPs, SIMPs, and Pure Higgsinos, Phys. Rev. Lett. 119, 131801 (2017).

[35] K. Bondarenko, A. Boyarsky, T. Bringmann, M. Hufnagel, K. Schmidt-Hoberg, and A. Sokolenko, Direct detection and complementary constraints for sub-GeV dark matter, J. High Energy Phys. 03 (2020) 118.

[36] A. Coogan, L. Morrison, and S. Profumo, Precision gammaray constraints for sub-GeV dark matter models, J. Cosmol. Astropart. Phys. 08 (2021) 044.

[37] V. Gluscevic and K. K. Boddy, Constraints on Scattering of $\mathrm{keV}-\mathrm{TeV}$ Dark Matter with Protons in the Early Universe, Phys. Rev. Lett. 121, 081301 (2018).

[38] W. L. Xu, C. Dvorkin, and A. Chael, Probing sub-GeV dark matter-baryon scattering with cosmological observables, Phys. Rev. D 97, 103530 (2018).

[39] T. R. Slatyer and C.-L. Wu, Early-universe constraints on dark matter-baryon scattering and their implications for a global 21 cm signal, Phys. Rev. D 98, 023013 (2018). 
[40] E. O. Nadler, V. Gluscevic, K. K. Boddy, and R. H. Wechsler, Constraints on dark matter microphysics from the Milky Way satellite population, Astrophys. J. Lett. 878, L32 (2019); 897, L46(E) (2020).

[41] K. Agashe, Y. Cui, L. Necib, and J. Thaler, (In)direct detection of boosted dark matter, J. Cosmol. Astropart. Phys. 10 (2014) 062.

[42] G. F. Giudice, D. Kim, J.-C. Park, and S. Shin, Inelastic boosted dark matter at direct detection experiments, Phys. Lett. B 780, 543 (2018).

[43] B. Fornal, P. Sandick, J. Shu, M. Su, and Y. Zhao, Boosted Dark Matter Interpretation of the XENON1T Excess, Phys. Rev. Lett. 125, 161804 (2020).

[44] C. V. Cappiello, K. C. Y. Ng, and J. F. Beacom, Reverse direct detection: Cosmic ray scattering with light dark matter, Phys. Rev. D 99, 063004 (2019).

[45] T. Bringmann and M. Pospelov, Novel Direct Detection Constraints on Light Dark Matter, Phys. Rev. Lett. 122, 171801 (2019).

[46] Y. Ema, F. Sala, and R. Sato, Light Dark Matter at Neutrino Experiments, Phys. Rev. Lett. 122, 181802 (2019).

[47] C. Cappiello and J. F. Beacom, Strong new limits on light dark matter from neutrino experiments, Phys. Rev. D 100, 103011 (2019).

[48] Y. Ema, F. Sala, and R. Sato, Neutrino experiments probe hadrophilic light dark matter, SciPost Phys. 10, 072 (2021).

[49] M. Andriamirado et al. (PROSPECT Collaboration), Limits on sub-GeV dark matter from the PROSPECT reactor antineutrino experiment, Phys. Rev. D 104, 012009 (2021).

[50] Y. B. Zel'dovich and I. D. Novikov, The hypothesis of cores retarded during expansion and the hot cosmological model, Sov. Astron. AJ (Engl. Transl.) 10, 602 (1967).

[51] E. R. Harrison, Fluctuations at the threshold of classical cosmology, Phys. Rev. D 1, 2726 (1970).

[52] S. W. Hawking, Black hole explosions?, Nature (London) 248, 30 (1974).

[53] S. W. Hawking, Particle creation by black holes, Commun. Math. Phys. 43, 199 (1975).

[54] I. B. Zeldovich, Charge asymmetry of the universe as a consequence of evaporation of black holes and of the asymmetry of the weak interaction, ZhETF Pisma Red. 24, 29 (1976).

[55] B. J. Carr, Some cosmological consequences of primordial black-hole evaporations, Astrophys. J. 206, 8 (1976).

[56] D. N. Page, Particle emission rates from a black hole: Massless particles from an uncharged, nonrotating hole, Phys. Rev. D 13, 198 (1976).

[57] D. N. Page, Particle emission rates from a black hole. 3. Charged leptons from a nonrotating hole, Phys. Rev. D 16, 2402 (1977).

[58] J. H. MacGibbon and B. R. Webber, Quark- and gluon-jet emission from primordial black holes: The instantaneous spectra, Phys. Rev. D 41, 3052 (1990).

[59] M. Boudaud and M. Cirelli, Voyager $1 e^{ \pm}$Further Constrain Primordial Black Holes as Dark Matter, Phys. Rev. Lett. 122, 041104 (2019).

[60] R. Laha, Primordial Black Holes as a Dark Matter Candidate are Severely Constrained by the Galactic Center $511 \mathrm{keV}$ $\gamma$-Ray Line, Phys. Rev. Lett. 123, 251101 (2019).

[61] G. Ballesteros, J. Coronado-Blázquez, and D. Gaggero, $\mathrm{X}$-ray and gamma-ray limits on the primordial black hole abundance from Hawking radiation, Phys. Lett. B 808, 135624 (2020).

[62] B. Dasgupta, R. Laha, and A. Ray, Neutrino and Positron Constraints on Spinning Primordial Black Hole Dark Matter, Phys. Rev. Lett. 125, 101101 (2020).

[63] A. Coogan, L. Morrison, and S. Profumo, Direct Detection of Hawking Radiation from Asteroid-Mass Primordial Black Holes, Phys. Rev. Lett. 126, 171101 (2021).

[64] R. Calabrese, D. F. G. Fiorillo, G. Miele, S. Morisi, and A. Palazzo, Primordial black hole dark matter evaporating on the neutrino floor, arXiv:2106.02492.

[65] V. De Romeri, P. Martínez-Miravé, and M. Tórtola, Signatures of primordial black hole dark matter at DUNE and THEIA, arXiv:2106.05013.

[66] M. J. Baker and A. Thamm, Probing the particle spectrum of nature with evaporating black holes, arXiv:2105.10506.

[67] B. Carr, K. Kohri, Y. Sendouda, and J. Yokoyama, Constraints on primordial black holes, arXiv:2002.12778.

[68] L. Morrison, S. Profumo, and Y. Yu, Melanopogenesis: Dark matter of (almost) any mass and baryonic matter from the evaporation of primordial black holes weighing a ton (or less), J. Cosmol. Astropart. Phys. 05 (2019) 005 .

[69] I. Baldes, Q. Decant, D. C. Hooper, and L. Lopez-Honorez, Non-cold dark matter from primordial black hole evaporation, J. Cosmol. Astropart. Phys. 08 (2020) 045.

[70] N. Bernal and Ó. Zapata, Self-interacting dark matter from primordial black holes, J. Cosmol. Astropart. Phys. 03 (2021) 007.

[71] N. Bernal and Ó. Zapata, Gravitational dark matter production: Primordial black holes and UV freeze-in, Phys. Lett. B 815, 136129 (2021).

[72] N. Bernal and Ó. Zapata, Dark matter in the time of primordial black holes, J. Cosmol. Astropart. Phys. 03 (2021) 015.

[73] J. Auffinger, I. Masina, and G. Orlando, Bounds on warm dark matter from Schwarzschild primordial black holes, Eur. Phys. J. Plus 136, 261 (2021).

[74] P. Gondolo, P. Sandick, and B. Shams Es Haghi, Effects of primordial black holes on dark matter models, Phys. Rev. D 102, 095018 (2020).

[75] I. Masina, Dark matter and dark radiation from evaporating Kerr primordial black holes, arXiv:2103.13825.

[76] A. Cheek, L. Heurtier, Y. F. Perez-Gonzalez, and J. Turner, Primordial black hole evaporation and dark matter production: I. Solely hawking radiation, arXiv:2107.00013 [Phys. Rev. D (to be published)].

[77] A. Cheek, L. Heurtier, Y. F. Perez-Gonzalez, and J. Turner, Primordial black hole evaporation and dark matter production: II. Interplay with the freeze-in/out mechanism, arXiv:2107.00016 [Phys. Rev. D (to be published)].

[78] A. Arbey and J. Auffinger, BlackHawk: A public code for calculating the Hawking evaporation spectra of any black hole distribution, Eur. Phys. J. C 79, 693 (2019).

[79] N. Aghanim et al. (Planck Collaboration), Planck 2018 results. VI. Cosmological parameters, Astron. Astrophys. 641, A6 (2020).

[80] J. F. Navarro, C. S. Frenk, and S. D. M. White, A universal density profile from hierarchical clustering, Astrophys. J. 490, 493 (1997). 
[81] G. D. Starkman, A. Gould, R. Esmailzadeh, and S. Dimopoulos, Opening the window on strongly interacting dark matter, Phys. Rev. D 41, 3594 (1990).

[82] G. D. Mack, J. F. Beacom, and G. Bertone, Towards closing the window on strongly interacting dark matter: Far-reaching constraints from earth's heat flow, Phys. Rev. D 76, 043523 (2007).

[83] B. J. Kavanagh, R. Catena, and C. Kouvaris, Signatures of earth-scattering in the direct detection of dark matter, J. Cosmol. Astropart. Phys. 01 (2017) 012.

[84] T. Emken and C. Kouvaris, How blind are underground and surface detectors to strongly interacting dark matter?, Phys. Rev. D 97, 115047 (2018).

[85] P. Capuano, G. De Luca, F. Di Sena, P. Gasparini, and R. Scarpa, The density of the rock covering Gran Sasso laboratories in central Apennines, Italy by underground gravity measurements, J. Appl. Geophys. 39, 25 (1998).

[86] R. H. Helm, Inelastic and elastic scattering of 187-Mev electrons from selected even-even nuclei, Phys. Rev. 104, 1466 (1956).

[87] J. D. Lewin and P. F. Smith, Review of mathematics, numerical factors, and corrections for dark matter experiments based on elastic nuclear recoil, Astropart. Phys. 6, 87 (1996).

[88] G. Tesic (VERITAS Collaboration), Searching for primordial black holes with the VERITAS gamma-ray experiment, J. Phys. Conf. Ser. 375, 052024 (2012).
[89] J.-F. Glicenstein, A. Barnacka, M. Vivier, and T. Herr (H.E.S.S. Collaboration), Limits on primordial black hole evaporation with the H.E.S.S. array of Cherenkov telescopes, in Proceedings of the 33rd International Cosmic Ray Conference (2013), 1307.4898.

[90] A. A. Abdo et al., Milagro limits and HAWC sensitivity for the rate-density of evaporating primordial black holes, Astropart. Phys. 64, 4 (2015).

[91] S. Archambault (VERITAS Collaboration), Search for primordial black hole evaporation with VERITAS, Proc. Sci. ICRC2017 (2018) 691 [arXiv:1709.00307].

[92] M. Ackermann et al. (Fermi-LAT Collaboration), Search for gamma-ray emission from local primordial black holes with the Fermi Large Area Telescope, Astrophys. J. 857, 49 (2018).

[93] A. Albert et al. (HAWC Collaboration), Constraining the local burst rate density of primordial black holes with HAWC, J. Cosmol. Astropart. Phys. 04 (2020) 026.

[94] P. Dave and I. Taboada (IceCube Collaboration), Neutrinos from primordial black hole evaporation, Proc. Sci. ICRC2019 (2021) 863 [arXiv:1908.05403].

[95] R. López-Coto, M. Doro, A. de Angelis, M. Mariotti, and J. P. Harding, Prospects for the observation of primordial black hole evaporation with the southern wide field of view gamma-ray observatory, J. Cosmol. Astropart. Phys. 08 (2021) 040. 